\title{
Treatment of generalized spacing with clear aligner therapy: Report of a case
}

\author{
Dr. Dashrath Kafle', Dr. Ravi Kumar Mahato², Dr. Abhisek Giri³ ${ }^{3}$ Dr. Sanjiv Luintel ${ }^{4}$, Dr. Arjun Karki ${ }^{4}$ \\ 'Professor and Head, ${ }^{2}$ Assistant Professor, ${ }^{3}$ Lecturer, ${ }^{4}$ Residents, \\ Department of Orthodontics, Dhulikhel Hospital, \\ Kathmandu University Teaching Hospital, Kavre, Nepal
}

Corresponding author:Dr Dashrath Kafle; Email: dashrath07@yahoo.com

\section{ABSTRACT}

Clear Aligners are becoming popular among patients in recent years because of its invisible nature, comfort to wear, less orthodontic emergencies and better hygiene control. However proper case selection is vital for the success. With the better understanding of aligner biomechanics and improvement in aligner materials and attachment design, more complex teeth movement is also possible with clear aligners. As there is always controversy on whether aligners cause tipping or bodily tooth movement, In this case report, a skeletal class I patient with generalized spacing in maxilla and mandible is presented which is treated effectively by bodily movement of incisors.

KEYWORDS: Clear Aligner, Mechanics, Orthodontics

\section{INTRODUCTION}

In the last two decades, there are marked leap forward in the field of orthodontics. The digital evolution has added significantly not only on diagnosis and treatment planning but also on treatment mechanics. The major advancements in the field of orthodontics are Digital radiographs, Digital cameras, Lab and Intraoral scanners, 3D printing and Metal milling. Clear aligner therapy was first started in 1999 with proprietary name INVISALIGN by Align technology has gained significant popularity since then. ${ }^{1}$ Extensive research on material science, improved software for virtual tooth movement and invisible nature of the system has allowed it to grow many folds in recent years. ${ }^{2}$ Though Invisalign from Align company still dominates the clear aligner market share worldwide, other clear aligner companies are also emerging. Recently In House Aligner(IHA) therapy is also getting popular in which orthodontists can fabricate the aligners of their patients by themselves using 3D printers, design software and vacuum formers. ${ }^{3}$ 3D printers are used more often in dentistry to fabricate temporary prosthesis, surgical guides and orthodontic models. Similarly newer companies are introducing intra oral scanners and lab scanners which might impact the growth of IHA in future. Clear aligners are invisible, comfortable and easy to wear. Moreover, compared with traditional fixed braces, clear aligners have less emergency appointments. ${ }^{2}$ Patient has the freedom to eat and maintaining oral hygiene is much better as it can be removed while eating and brushing. Though there are various claims about its efficacy, there is no denial that Clear Aligners are going to stay in orthodontic profession for long. The treatment mechanics with aligners is getting better with time. Here we present a case report done with clear aligner.

\section{CASE REPORT}

A 22 years old patient came to Department of Orthodontics, Dhulikhel Hospital, Kathmandu University Teaching Hospital with chief complain of wide spacing between upper and lower teeth. On examination she had generalized spacing of maxillary and mandibular dentition. Her molar and canine were class I relationship. However her upper left second premolar and first molar were in cross bite relation with lower left second premolar and first molar. She did not have any tongue thrust habit and her frenal attachment was normal. Her cephalometric values show her to be the case of 
skeletal class I jaw base with $5 \mathrm{~mm}$ and $6 \mathrm{~mm}$ spacing in maxilla and mandible respectively associated with posterior cross bite(left). Orthopantomogram x-ray shows presence of all the 32 teeth. No any other defects were found in temporomandibular joint and surrounding structures. So she was diagnosed with the case of skeletal Class I malocclusion with spacing and left sided cross bite.

After discussing different treatment plan with patient, she choose Clear Aligner Therapy (SmartAlign, Smart3D Pvt Ltd, Kathmandu, Nepal) for her treatment as patient was frequent traveler and regular follow up was not possible. After rubber base impression is taken by two step technique, impression was sent to lab. The impression was scanned with labscanner(E1, 3Shape, Denmark) after pouring cast with die stone. The virtual planning was made by one of the author with 3Shape Aligner Studio software (3Shape, Denmark). While designing the tooth movenet, it was important to move the tooth bodily because of large interdental spacing.
Attachments were designed as necessary to move the teeth bodily. Approximately $4 \mathrm{~mm}$ of mesio-distal movement was necessary for the complete closure of spacing. Some amount of over correction was added in all the planes for better finish of the case. (Fig 3 )

Total number of aligners needed to complete this treatment were 19 followed by 10 refinement sets. The end treatment photograph shows nicely closed space, Class I Molar, Incisor and Canine relation. post treatment cephalograph and orthopantomograph shows translator movement of teeth with no signs of external apical root resorption. Cephalometric superimposition shows improvement in incisor angulation and facial profile (Fig 6). The posterior crossbite in relation to 26 and 36 has not been corrected completely though. Patient was given lower bonded retainer and upper bonded and vacuum pressed retainer (Fig 7). Retainer protocol was: night time wear of clear retainer for 1 year followed by gradual tapering of retainer. Patient is encouraged to wear bonded retainer for long term.
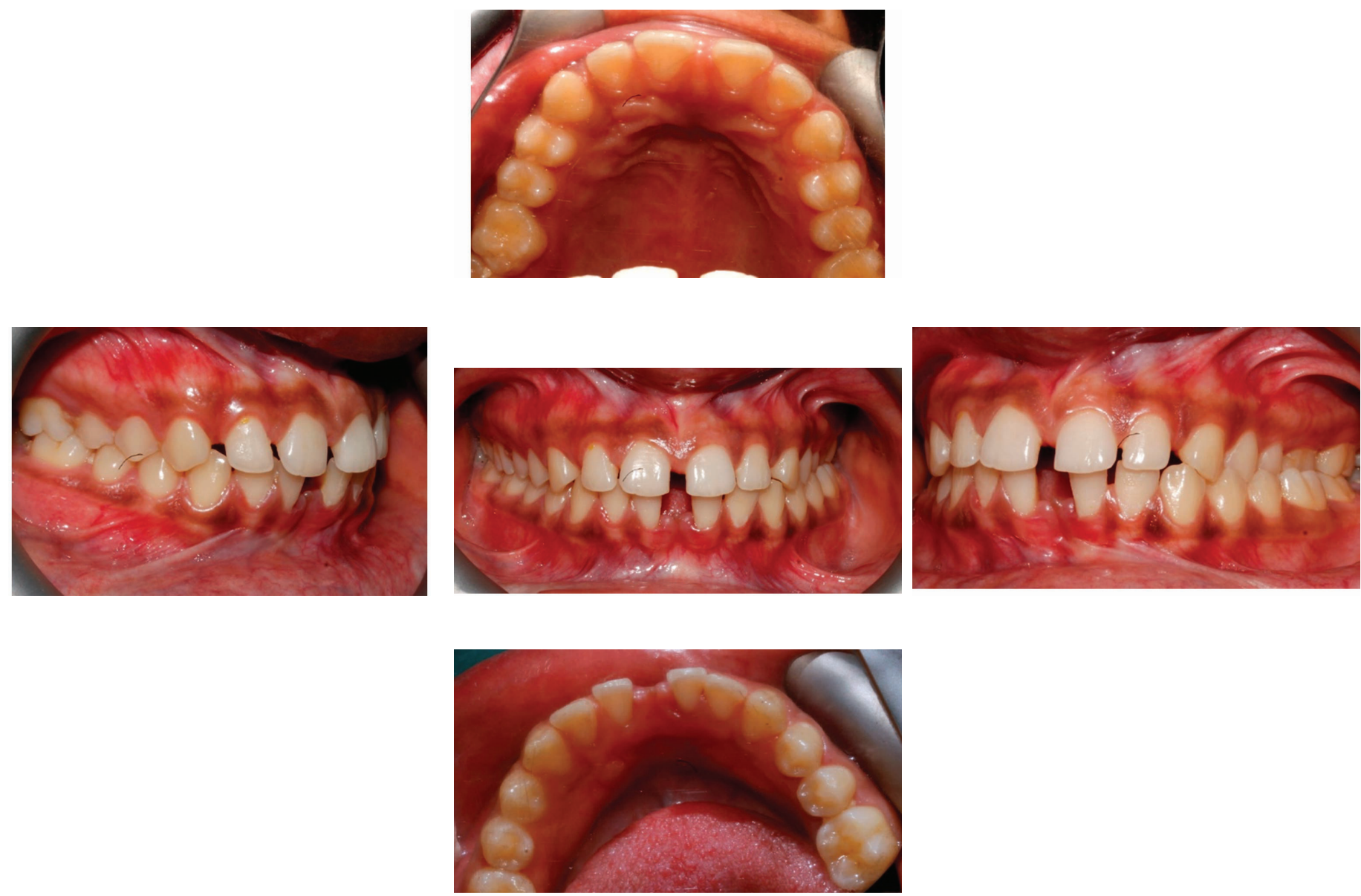

Fig 1: Pre Treatment Intraoral photograph 

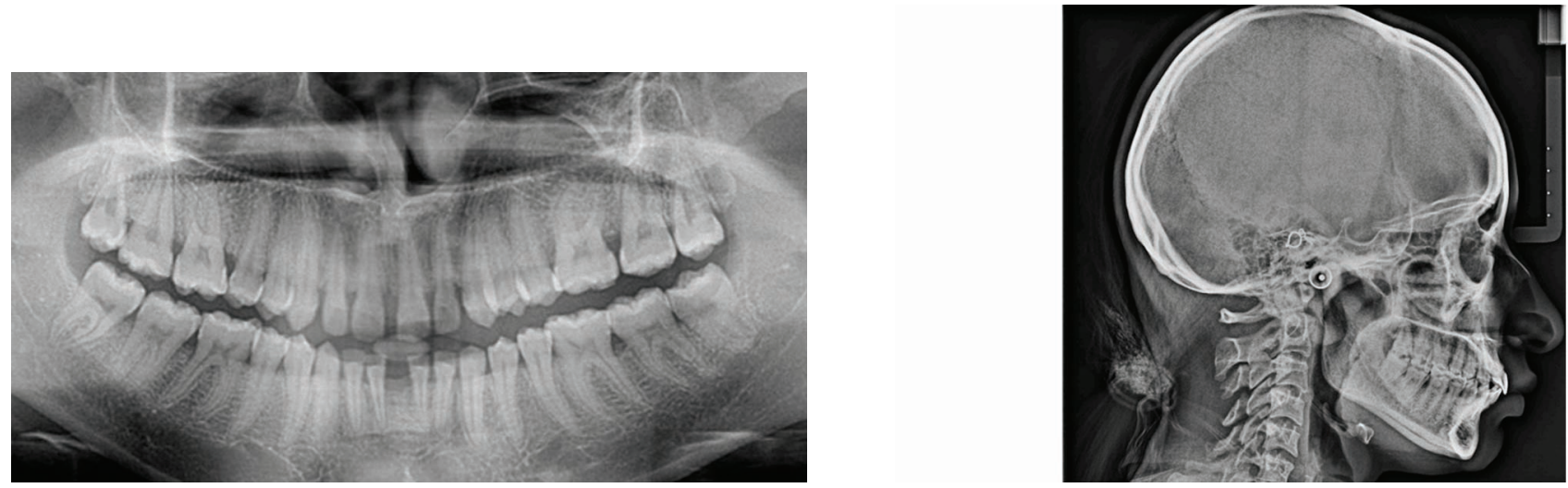

Fig 2: Pre Treatment Radiographs
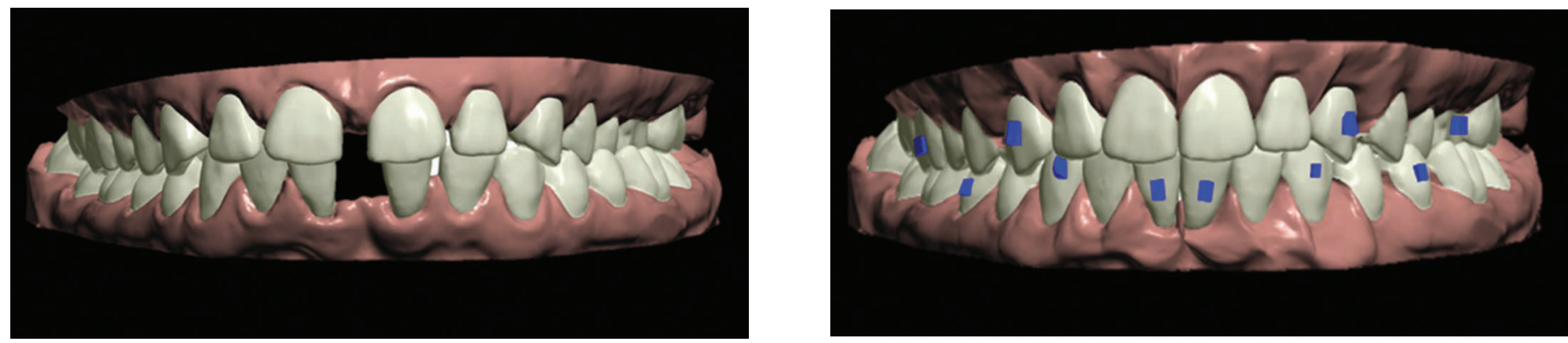

Fig 3: Virtual Treatment Planning
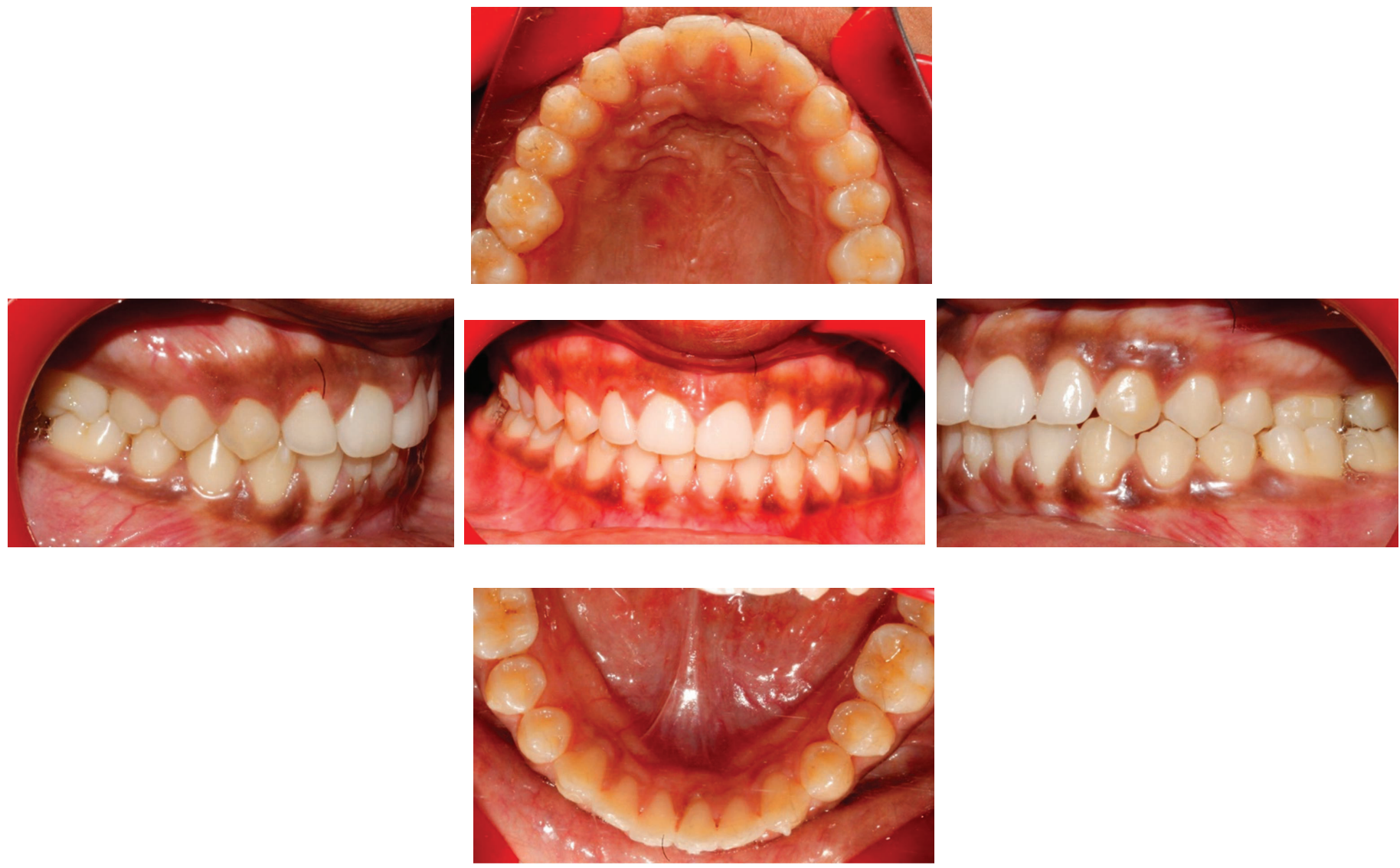

Fig: 4: Post Treatment Intraoral Photograph 

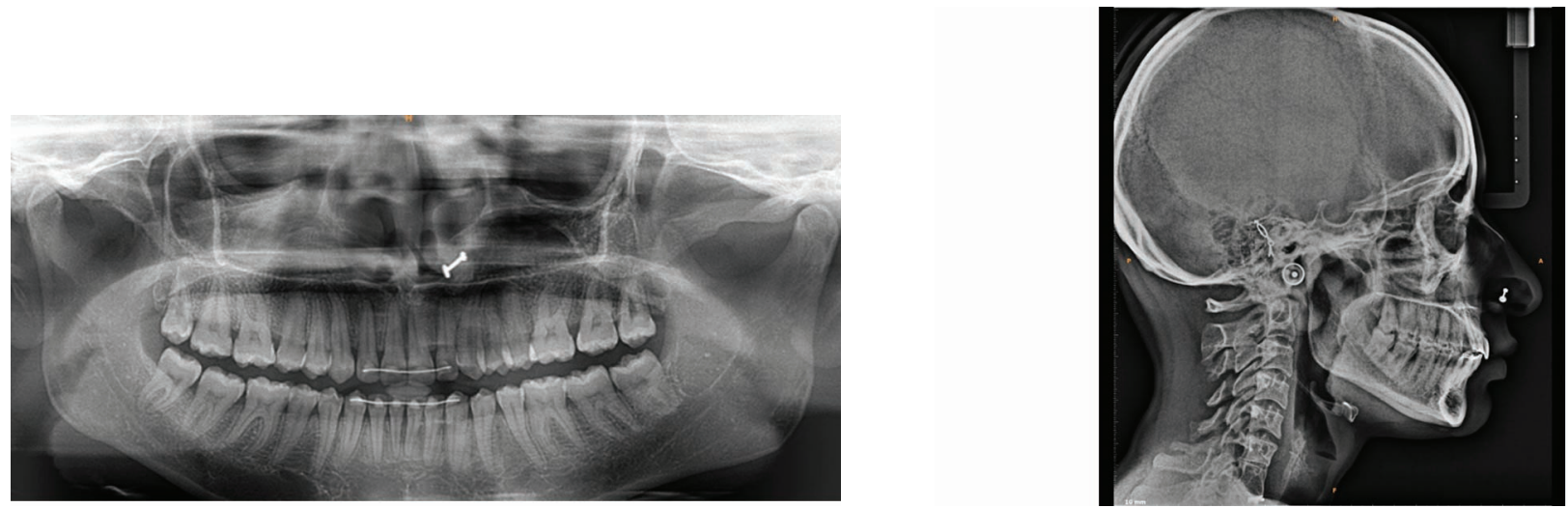

Fig 5: Post Treatment radiographs

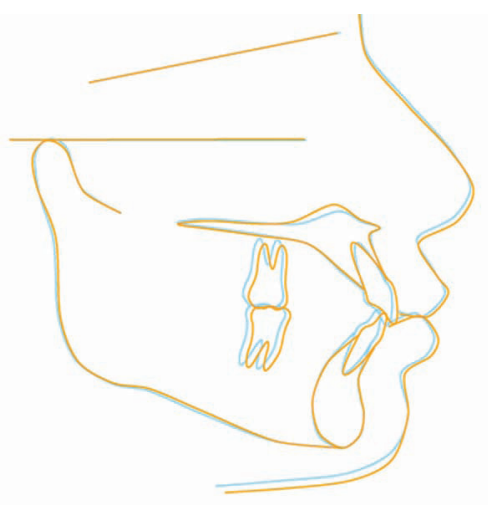

Fig 6: Superimposition
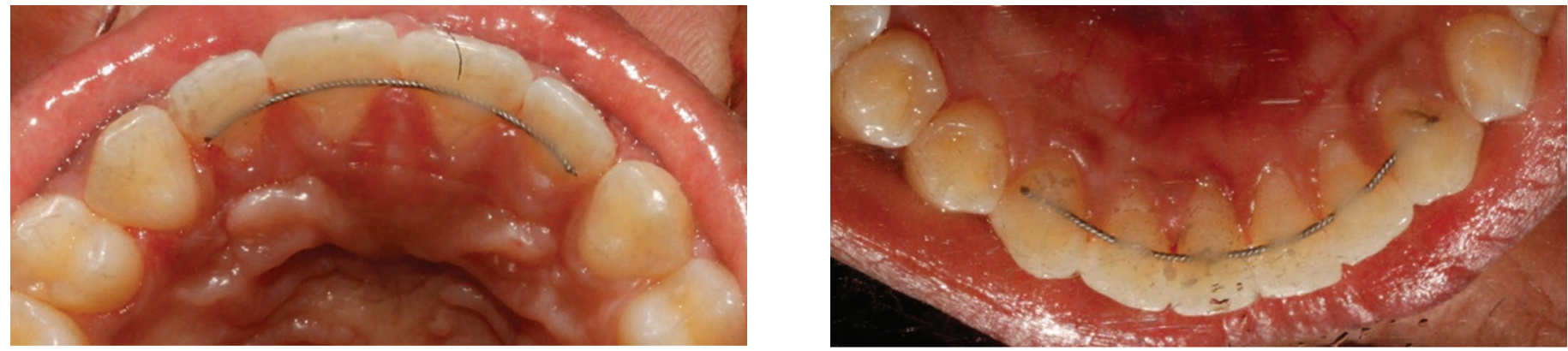

Fig 7: Retention Photograph

\section{DISCUSSION}

Though there are numerous controversies regarding the efficacy of clear aligner therapy, in this case report, the generalized spacing seem to be nicely treated. Antero-Posterior and Mesio-Distal movement is predicatble with clear aligners. ${ }^{4}$ Rotations more than 45 degree are difficult to correct with aligners alone. Moreover posterior rotations are difficult to treat than anteriors. ${ }^{5}$ Transverse correction in this case was not achieved fully. Deep bite correction as well as open bite corrections are regarded as one of the difficult malocclusion to correct. ${ }^{2,6-9}$
Clear aligners are thought to move the teeth by tipping. In this case, the comparision of pre and post treatment panoramic $x$-ray shows bodily movement of the incisors. Various attachments are used to control the tooth momevemt in clear aligner therapy. ${ }^{6}$ In this case right and left rotational control attachments were used in incisors whereas horizontal attachments were used on molars. The major disadvantage of the clear aligner therapy is patient compliance. ${ }^{10,11}$ Our patient was very complaint and self-motivated for clear aligner therapy. For about 9 months, remote monitoring was only possible. However patient wore aligners as prescribed 
by the treating doctor. Most of the clear aligner cases need refinement steps to obtain the desired outcome. In this case, number of refinement aligners were 10 and 12 in maxilla and mandible respectively. There are some recent studies which have shown that bodily movement is possible with clear aligners and they are as effective as fixed orthodontic therapy. ${ }^{12,13}$ However we should be very caution while selecting the cases in clear aligner therapy as the final outcome depends upon the virtual planning, aligner material, attachment designs, patient compliance and many other factors.

\section{CONCLUSION}

Clear Aligners as well as In House Aligner (IHA) can be viable alternative to traditional orthodontic therapies. However proper case selection is very crucial for gaining success with this new system. Advanced training on this new technology should helporthodontists for better case selection and treatment execution.

\section{REFERENCES}

1. Gierie WV. Clear aligner therapy: An overview. J Clin Orthod 2018;52(12):665-74.

2. Yassir YA, Nabbat SA, McIntyre GT, Bearn DR. Clinical effectiveness of clear aligner treatment compared to fixed appliance treatment: an overview of systematic reviews. Clin Oral Investig 2022.

3. Sachdev S, Tantidhnazet S, Saengfai NN. Accuracy of Tooth Movement with In-House Clear Aligners. J World Fed Orthod 2021;10(4):177-82.

4. Ben Gassem AA. Does Clear Aligner Treatment Result in Different Patient Perceptions of Treatment Process and Outcomes Compared to Conventional/Traditional Fixed Appliance Treatment: A Literature Review. Eur J Dent 2021.

5. Robertson L, Kaur H, Fagundes NCF, Romanyk D, Major P, Flores Mir C. Effectiveness of clear aligner therapy for orthodontic treatment: A systematic review. Orthod Craniofac Res 2020;23(2):133-42.

6. Ahmed T, Padmanabhan S, Pottipalli Sathyanarayana H. Effects of varying attachment positions on palatal displacement of maxillary incisors with clear aligner therapy : A three-dimensional finite element analysis. J Orofac Orthop 2022.

7. Wu D, Zhao Y, Ma M, Zhang Q, Lei H, Wang Y, et al. Efficacy of mandibular molar distalization by clear aligner treatment. Zhong Nan Da Xue Xue Bao Yi Xue Ban 2021;46(10):1114-21.

8. d'Apuzzo F, Perillo L, Carrico CK, Castroflorio T, Grassia V, Lindauer SJ, et al. Clear aligner treatment: different perspectives between orthodontists and general dentists. Prog Orthod 2019;20(1):10.

9. Kim TW, Echarri P. Clear aligner. an efficient, esthetic, and comfortable option for an adult patient. World J Orthod 2007;8(1):13-8.

10. Crouse JM. Patient compliance with removable clear aligner therapy. J Clin Orthod 2018;52(12):710-13.

11. Grunheid T, Gaalaas S, Hamdan H, Larson BE. Effect of clear aligner therapy on the buccolingual inclination of mandibular canines and the intercanine distance. Angle Orthod 2016;86(1):10-6.

12. Tepedino $M$, Paoloni V, Cozza P, Chimenti C. Movement of anterior teeth using clear aligners: a three-dimensional, retrospective evaluation. Prog Orthod 2018;19(1):9.

13. Jiang T, Jiang Y-N, Chu F-T, Lu P-J, Tang G-H. A cone-beam computed tomographic study evaluating the efficacy of incisor movement with clear aligners: Assessment of incisor pure tipping, controlled tipping, translation, and torque. American Journal of Orthodontics and Dentofacial Orthopedics 2021;159(5):635-43. 